\title{
Syncytia Induction by Clinical Isolates of Human Respiratory Syncytial Virus A
}

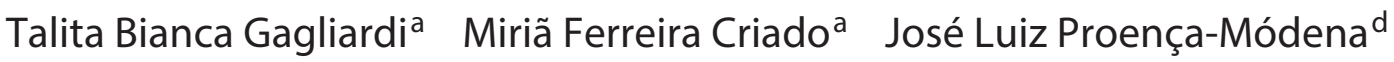

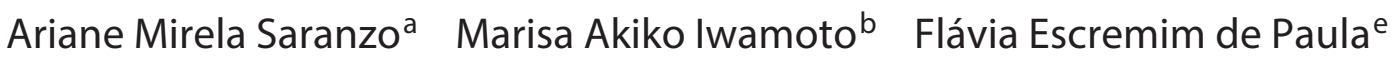 \\ Ricardo Souza Cardoso ${ }^{a}$ Luana Sela Delcaro ${ }^{a}$ Maria Lúcia Silva ${ }^{a}$ \\ Ataide Ademir Câmara ${ }^{c}$ Eurico Arruda ${ }^{a}$ \\ ${ }^{a}$ Department of Cell Biology, University of São Paulo School of Medicine, ${ }^{\mathrm{b}}$ Barão de Mauá University, and \\ 'University of Ribeirão Preto (UNAERP) School of Medicine, Ribeirão Preto, dDepartment of Genetics, Evolution and \\ Bioagents, Institute of Biology, State University of Campinas, Campinas, and ${ }^{\mathrm{e} M o l e c u l a r}$ Oncology Research Center, \\ Barretos Cancer Hospital, Barretos, Brazil
}

\section{Keywords}

Respiratory syncytial virus · Respiratory syncytial virus clinical isolates - Syncytia quantification · HRSV F protein

\begin{abstract}
Objective: Syncytia formation is the hallmark of the cytopathic effect caused by human respiratory syncytial virus (HRSV), which is the most important viral respiratory pathogen in children. This article reports methodological improvements in primary HRSV isolation and the importance of syncytia formation and $m$ RNA levels of $F$ protein for the progeny yield, using clinical isolates of HRSV. Methods: The A and B strains of HRSV were isolated in HEp-2 cell cultures from fresh and frozen nasopharyngeal aspirates. The formation of syncytia was evaluated using 2 different assays. Levels of $F$ protein mRNA were quantified by real-time PCR while HRSV progeny titration was done by plaque assay. Results: HRSV was primarily isolated from 238 of 312 (90.7\%) samples, and 13 of these (12 HRSV-A and 1 HRSV-B) were continuously passaged in vitro. The quantity and size of syncytia formed
\end{abstract}

\section{KARGER}

(C) 2017 S. Karger AG, Basel

E-Mail karger@karger.com

www.karger.com/int by 6 pure HRSV-A clinical isolates were different, as were the levels of $F$ protein mRNA. Conclusion: There is a direct correlation of quantities of syncytia and inoculum size, but not with mRNA levels of HRSV-A F protein. Importantly, levels of $\mathrm{F}$ protein $\mathrm{mRNA}$ were directly related to progeny production.

(c) 2017 S. Karger AG, Basel

Human respiratory syncytial virus (HRSV), a member of the family Pneumoviridae, has a 15.2-kb, negativesense, single-strand RNA genome that encodes 11 proteins. The fusion protein $(\mathrm{F})$ promotes fusion between virus envelope and cell membrane, which is essential for virus entry. This protein also promotes the fusion of plasma membranes between adjacent cells, resulting in a giant cell with $>3$ nuclei, the syncytium [1]. This cytopathic effect induced by HRSV is considered important for virus spread along epithelial surfaces, thus minimizing virus exposure to extracellular components of the immune response. Several cellular and viral factors partici- 
pate in syncytia formation, such as Rho A protein and the viral attachment $(\mathrm{G})$ and small hydrophobic $(\mathrm{SH})$ envelope proteins, but the $\mathrm{F}$ protein is the only one regarded as essential for membrane fusion [2-5].

Most of the published studies on HRSV have been done with extensively passaged laboratory prototype strains that have been adapted for propagation in continuous cell lines, such as the strains Long (ATCC ${ }^{\circledR}$ VR$26 \mathrm{D}^{\mathrm{TM}}$ ) and $\mathrm{A} 2$ [6-8]. But the serial passaging has selected HRSV laboratory strains with significant mutations in their genomes, which may result in important changes in virus-host interactions compared to wild-type HRSV strains [1]. Despite this, clinical strains of HRSV are rarely used in studies, due mainly to the difficulty of obtaining primary isolates. A previous comparison between continuous cell lines for the recovery of HRSV from fresh samples revealed that HEp-2 cells enabled higher rates of isolation from fresh samples [9]. Taking this into consideration, this report undertook to describe efforts to improve the recovery of HRSV primary isolates, resulting in the establishment of a protocol that led to the acquisition of several clinical HRSV isolates amenable to be passaged in vitro to prepare stocks. Propagable clinical HRSV-A strains shed by patients without other respiratory virus coinfections were assessed for their ability to form syncytia and the link to the production of messenger RNA (mRNA) for the F protein and progeny yield.

This study was approved by the Internal Review Board of the University of São Paulo Hospital (protocol No. HCRP A07-020). Nasopharyngeal aspirates (NPAs) were collected from children $<5$ years of age, after obtaining parental consent. Patients were seen at the following participating health care facilities: the Primary Care Center of the University of Sao Paulo School of Medicine and the São Lucas Hospital, both located in the city of Ribeirão Preto, SP, Brazil. The patients had with acute respiratory symptoms, including cough, coryza, sneezing, fever, wheezing, and stuffy nose. The NPAs were sent to the laboratory on ice, where they were processed within $<3 \mathrm{~h}$ after collection (online suppl. material; for all online suppl. material, see www.karger.com/doi/10.1159/000480014).

To provide pediatricians with the results of the HRSV screening, NPAs were routinely tested by the rapid assay "BD Directigen ${ }^{\mathrm{TM}}$ EZ RSV" (Becton Dickinson, Frank Lakes, NJ, USA), or by a previously published RT-PCR assay developed in-house [10]. Primary HRSV isolation (P1) was done in HEp-2 cell cultures inoculated with HRSV-positive NPAs, either freshly collected or kept frozen for 1 or 2 years. To make viral stocks, 2 or 3 additional passages ( $\mathrm{P} 3$ and $\mathrm{P} 4$ ) were also done in fresh monolay-

Syncytia Formation by HRSV Clinical Isolates ers of HEp-2 cells. All viral passages (P1-P4) were tested for HRSV by indirect immunofluorescence using specific antibody MAB858-4 (Chemicon International Inc., Temecula, CA, USA) (online suppl. material).

To avoid the interference of another virus in the syncytia assays, the NPAs, from which clinical HRSV were isolated, were tested for other respiratory viruses, according to a previously published protocol [10]. Primers specific for human metapneumovirus (HMPV), human parainfluenza (HPIV), human influenza virus (flu), human rhinovirus (RV), human coronaviruses $229 \mathrm{E}$ and OC43 (HCoV), human adenovirus (HAdV), and human bocavirus (HBoV) were used in these assays [10]. Additionally, the pure propagable clinical HRSV isolates (P3 or $\mathrm{P} 4$ ) were classified as belonging to the A or B group by real-time PCR. This assay was done using the TaqMan strategy (TaqMan ${ }^{\circledR}$ Universal PCR Master Mix, Applied Biosystems, Sunnyvale, CA, USA), with specific primers/ probes for the HRSV N gene (online suppl. material).

Two different methods were used to assess syncytia induction in vitro by clinical HRSV strains: a manual count of syncytia in HEp-2 monolayers and flow cytometry. The visualization and counting of syncytia assay (CSA) were done with semiconfluent (70\%) HEp-2 cell monolayers seeded onto $13-\mathrm{mm}$ coverslips, followed by Giemsa staining. Syncytia were also quantified by a flow cytometry syncytia assay (FCSA) using DNA staining with propidium iodide, based on a previously published protocol described for HIV [11] (online suppl. material).

F-protein mRNA and the genome of HRSV were quantitated by real-time PCR (qPCR) with SYBR ${ }^{\circledR}$ Green PCR Master Mix (Applied Biosystems). qPCR for the HRSV genome was done with primers targeting sequences of leader/NS1 genes, and the results were analyzed using 7300 System software (Applied Biosystems. HRSV progeny titers were determined in duplicate by plaque assay using a previously published protocol [12] (online suppl. material).

The protocol described here to isolate HRSV was applied to 312 NPAs, which were previously known to be positive for HRSV by at least one of the methods (rapid test and RT-PCR). Primary HRSV isolates were obtained from $86 \%(74 / 86)$ of fresh NPAs, $81.2 \%(95 / 117)$ of the NPAs that had been frozen at $-70^{\circ} \mathrm{C}$ for 1 year, and $63.3 \%$ $(69 / 109)$ of the NPAs that had been frozen for 2 years. Of 238 primary HRSV isolates, only 13 (5.5\%) could be successfully propagated in HEp-2 cells, and they originated from 12 subgroup A and 1 subgroup B HRSV propagable clinical stocks. One of the 12 propagable HRSV-A isolates was obtained from an NPA frozen for 2 years, 4 were from

Intervirology 2017;60:56-60 DOI: $10.1159 / 000480014$ 
NPAs frozen for 1 year, and the remaining 8 , including the only HRSV-B stock, were obtained from fresh NPAs. Importantly, if no cytopathic effect was seen in P2 and if immunofluorescence was negative, HRSV propagation by further passages was considered unsuccessful.

To evaluate the ability of clinical HRSV isolates to form syncytia, all NPAs from which HRSV was successfully recovered, were retrospectively tested for the other common respiratory viruses. Of the 13 propagable clinical HRSV strains obtained, 6 were found to have come from NPAs which were also positive for other viruses: 2 for HPIV-2, 2 for HMPV, 1 for human adenovirus HAdV, and 1 for HPIV-2 and HAdV simultaneously. All propagable HRSVs recovered from NPAs that were also positive for other coinfecting viruses were excluded from subsequent syncytia formation analyses. Of the 7 pure clinical HRSV strains, 1 was of group B (HRSV97). Therefore, the subsequent analyses were done with the 6 pure HRSV strains of group A.

Two different syncytia formation assays (CSA and FCSA) were used in this study. For the CSA, 24-well-plate HEp- 2 cell cultures were infected with the 6 pure HRSVA isolates. The CSA protocol is described in the online supplementary material. The quantity of syncytia observed in this assay varied from 763 (HRSV191) to 3,344 (HRSV66) syncytia per coverslip; while the size of the syncytia varied from 4 (HRSV69) to 9 (HRSV143) nuclei per syncytium (data not shown). A similar variation in quantities of syncytia was observed on FCSA: from 22 (HRSV66) to 114 (HRSV105) syncytia per 1,000 cells (data not shown). Because FCSA was more sensitive and accurate than CSA to quantitate syncytia, subsequent analyses were done based on the FCSA results. On the other hand, since FCSA does not allow for determination of quantities of nuclei per syncytium, the CSA results were used for this purpose.

Genomes from 6 pure HRSV-A isolates were quantitated in triplicate by qPCR targeting the leader/NS1 genes. The quantities of HRSV RNA copies in the inocula were normalized by the number of cells in the cultures, and this adjusted number was considered as a molecular equivalent of HRSV inocula titration. Comparing results from syncytia assays and HRSV inocula, a direct correlation became apparent between inoculum size and the quantity of syncytia induced by 5 of the 6 clinical HRSV-A isolates (Fig. 1a). However, no relation was observed between quantities of inocula and sizes of syncytia (Fig. 1b). Because the F protein of HRSV is such an important factor in syncytia formation $[1-5,10]$, a quantitation of the mRNA for the F protein was carried out in triplicate for the 6 pure HRSV-A strains. The geometric means of qPCR results for mRNA of the F protein were normalized by molecular inoculum size. The quantities of F-protein mRNA varied significantly across the 6 clinical HRSV-A isolates: from 3,576 (HRSV66) to 2,898,901 (HRSV69) mRNA molecules per copy of HRSV genome in the inoculum (Fig. 1c). Importantly, the normalized quantities of $\mathrm{F}$ protein mRNA showed no apparent correlation with numbers or sizes of syncytia (data not shown).

The progeny yields in HEp- 2 cells infected with 6 pure clinical HRSV-A strains were determined by plaque assay at $24 \mathrm{~h}$ after infection (following the protocol detailed in the online suppl. material), and the results were normalized according to molecular inoculum size. Progeny titers varied from 2 (HRSV191) to 110,294 (HRSV205) PFU/ $\mathrm{mL} /$ copy of inoculum RNA (data not shown). Except for 1 strain (HRSV191), there was a direct, yet not linear, correlation between quantities of mRNA for the F protein and progeny production (Fig. 1d).

The strategy of exposing HEp-2 cell cultures to low $\mathrm{pH}$ not only improved the primary HRSV isolation from fresh human NPAs, but also from the frozen samples. The rates of HRSV isolation reported here are higher than what has been reported by other study groups $[13,14]$. The approach of lowering $\mathrm{pH}$ in order to isolate HRSV from clinical samples is based on a study published in 2006 that showed enhancement of fusion mediated by the F protein of human metapneumovirus in LLCMK2 cells by previous treatment with a low $\mathrm{pH}$ [15]. The reason why this preincubation of cells at a low $\mathrm{pH}$ improved primary HRSV isolation is unknown. However, a preliminary experiment done with the HRSV strain Long in HEp-2 cells showed that exposure of cells to a pH of 5 for 30 min before HRSV infection led to a 2-fold enhancement of the quantity, but not the size, of the syncytia when compared to controls kept at a $\mathrm{pH}$ of 7 (unpubl. observ.). Despite this observed high rate of primary HRSV isolation, the ability to produce stocks from propagable clinical HRSV strains was low (5.5\%), even using the same protocol. The reasons for this are still unclear, but maybe there are differences between HRSV particles shed in the context of human respiratory secretions, as to the susceptibility of the F protein to cleavage. In this regard, a reduced susceptibility of the HRSV F protein to cleavage by furin has been associated with lower $\mathrm{F}$ protein fusogenic activity in persistently infected murine macrophages [16]. Taking this into consideration, it would be tempting to suggest that components of human respiratory secretions present in the clinical sample may work as adjuvants of HRSV infectivity and facilitate the recovery of primary
58

Intervirology 2017;60:56-60

DOI: $10.1159 / 000480014$
Gagliardi et al. 

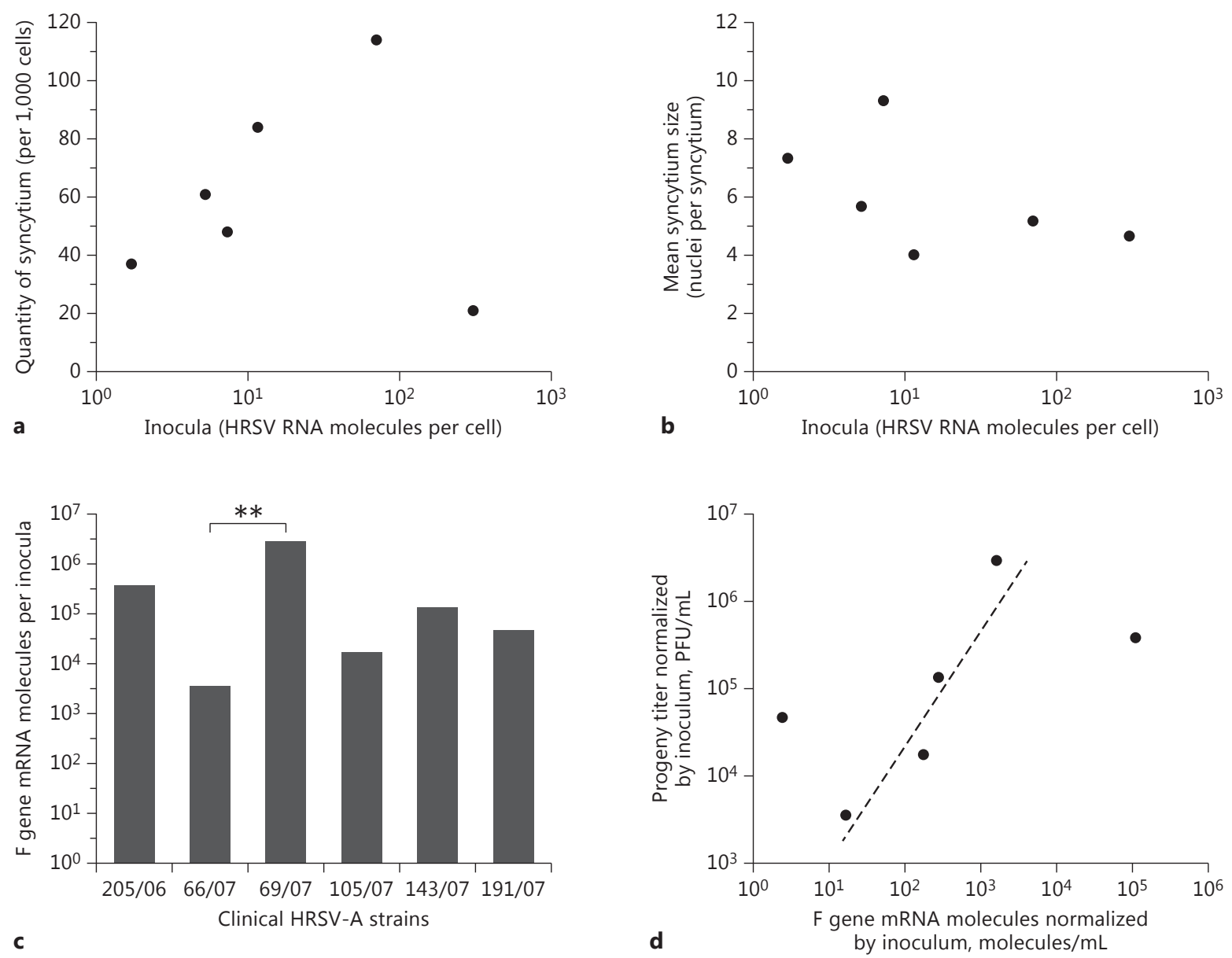

Fig. 1. Formation of syncytia, quantity of $\mathrm{F}$ protein mRNA and progeny yield of 6 clinical HRSV-A isolates in HEp-2 cells. a Relation between quantity of syncytia and inoculum size. b Relation between sizes of syncytia (expressed as number of nuclei per syncytium) and inoculum size. c Relative quantity of mRNA for the F

protein normalized by inoculum size for $6 \mathrm{HRSV}$-A clinical strains (ANOVA statistical analysis; ${ }^{* *} p<0.01$ ). d Relation between relative quantity of mRNA for the F protein (normalized by inoculum size) and progeny production.

isolates in the cell culture compared to subsequent passages in the cell culture. Although the rate of acquisition of 5.5\% propagable HRSV isolates was not high, it is higher than we have had before, and, to the best of our knowledge, higher than what has been reported in the literature $[13,14]$. Importantly, 13 clinical HRSV stocks were obtained in this study, and these can be used in future studies in vitro or in vivo, since they are amenable to propagation in continuous cell lines, even after freezing/thawing.

This study also helped to establish 2 simple methods to quantitate and measure syncytia. The simple microscopic examination and syncytia counting on stained coverslips (the CSA) have the advantage of being inex-

pensive, and require only an optical microscope and a minimally trained observer. Flow cytometry (the FSCA) has the benefit of being observer-independent, but it does not allow for determination of syncytium size.

As expected, the ability of 6 pure clinical HRSV-A strains to induce syncytia was directly related to the inoculum size, as determined by qPCR (Fig. 1a).

The relative quantity of mRNA for the HRSV F protein varied extensively over a range of several orders of magnitude in 6 pure clinical HRSV-A strains, even when the numbers were corrected for inoculum size. To the best of our knowledge, this is a novel observation, demonstrating for the first time that the abundance of F-protein gene 
transcripts may vary significantly between HRSV-A clinical isolates in the same culture conditions. Surprisingly, there was no correlation of relative quantity of F-protein mRNA with the number or size of the syncytia. However, it should be kept in mind that, in addition to the unquestionable role of $F$ protein, other viral proteins, such as $G$ and $\mathrm{SH}$, play important roles in the spread of HRSV from cell to cell $[4,5]$, and also that the mRNAs for the G and $\mathrm{SH}$ proteins were not quantitated in this study. Additionally, the cleavage of the protein precursor F0 by host-cell furin is an important event regulating F-protein fusional activity [16], and it is reasonable to speculate that F proteins from different HRSV strains may display variable susceptibility to cleavage by furin and, hence, different syncytium induction.
This study shows for the first time that quantities of Fprotein mRNA produced by the infection of HEp-2 cells with clinical HRSV strains may be related to progeny production. Ongoing efforts to obtain more propagable clinical HRSV isolates will enable future studies to be conducted that address the strain-dependent differences in the pathogenesis of this important agent.

\section{Acknowledgements}

We thank the São Paulo State Research Foundation (FAPESP) and the Brazilian National Research Council (CNPq) for financial support. We also thank the Biostatistics Service of the University of São Paulo School of Medicine in Ribeirão Preto, for the skilled help, and Gustavo Olszanski Acrani for helpful discussion.

\section{References}

1 Collins P, Karron RA: Respiratory syncytial virus and metapneumovirus; in Knipe DM, Howley PM (eds): Fields Virology. Philadelphia, Lippincott Williams \& Wilkins, 2013, vol 1, pp 1086-1123.

2 Gower TL, Pastey MK, Peeples ME, Collins PL, McCurdy LH, Hart TK, Guth A, Johnson TR, Graham BS: RhoA signaling is required for respiratory syncytial virus-induced syncytium formation and filamentous virion morphology. J Virol 2005;79:5326-5336.

3 Karron RA, Buonagurio DA, Georgiu AF: Respiratory syncytial virus (RSV) SH and G proteins are not essential for viral replication in vitro: Clinical evaluation and molecular characterization of a cold-passaged, attenuated RSV subgroup B mutant. Proc Natl Acad Sci USA 1997;94:13961-13966.

4 Bukreyev A, Whitehead SS, Murphy BR, Collins PL: Recombinant respiratory syncytial virus from which the entire $\mathrm{SH}$ gene has been deleted grows efficiently in cell culture and exhibits site-specific attenuation in the respiratory tract of the mouse. J Virol 1997;71: 8973-8982.

5 Heminway BR, Yu Y, Tanaka Y, Perrine KG, Gustafson E, Bernstein JM, Galinski MS: Analysis of respiratory syncytial virus F, G, and SH proteins in cell fusion. Virology 1994; 200:801-805.
6 Lewis FA, Rae MI, Lehmann NI, Ferris AA: A syncytial virus associated with epidemic disease of the lower respiratory tract in infants and young children. Med J Aust 1961;2:932933.

7 Rameix-Welti MA, Le Goffic R, Hervé PL, Sourimant J, Rémot A, Riffault S, Yu Q, Galloux M, Gault E, Eléouët JF: Visualizing the replication of respiratory syncytial virus in cells and in living mice. Nat Commun 2014;5: 5104.

8 Lemon K, Nguyen DT, Ludlow M, Rennick MJ, Yüksel S, van Amerongen G, McQuaid S, Rima BK, de Swart RL, Duprex WP: Recombinant subgroup B human respiratory syncytial virus expressing enhanced green fluorescent protein efficiently replicates in primary human cells and is virulent in cotton rats. J Virol 2015;89:2849-2856.

9 Perini AP, Barbosa ML, Botosso VF, Moraes CTP, Gillio AE, Hens N, Stewien KE, Durigon EL: Comparison of HeLa-I, Hep-2 and NCIH292 cell lines for the isolation of human respiratory syncytial virus (HRSV). J Virol Methods 2007;146:368-371.

10 Gagliardi TB, De Paula FE, Iwamoto MA, Proenca-Modena JL, Santos AE, Camara AA, Cervi MC, Cintra OA, Arruda E: Concurrent detection of other respiratory viruses in children shedding viable human respiratory syncytial virus. J Med Virol 2013;85:1852-1859.
11 Wunschmann S, Stapleton JT: Fluorescencebased quantitative methods for detecting human immunodeficiency virus type 1-induced syncytia. J Clin Microbiol 2000;38:30553060.

12 McKimm-Breschkin JL: A simplified plaque assay for respiratory syncytial virus-direct visualization of plaques without immunostaining. J Virol Methods 2004;120:113-117.

13 Lawlor HA, Schickli JH, Tang RS: A single amino acid in the F2 subunit of respiratory syncytial virus fusion protein alters growth and fusogenicity. J Gen Virol 2013;94:26272635.

14 Thevenin T, Lobert PE, Dewilde A, Hober D: A respiratory syncytial virus isolate enables the testing of virucidal products. Int J Hyg Environ Health 2011:215:293-296.

15 Schowalter RM, Smith SE, Dutch ER: Characterization of human metapneumovirus F protein-promoted membrane fusion: critical roles for proteolytic processing and low $\mathrm{pH}$. J Virol 2006;80:10931-10941.

16 Sarmiento RE, Arias CF, Méndez E, Gómez B: Characterization of a persistent respiratory syncytial virus showing a low-fusogenic activity associated to an impaired F protein. Virus Res 2009;139:39-47. 